\title{
Editorial
}

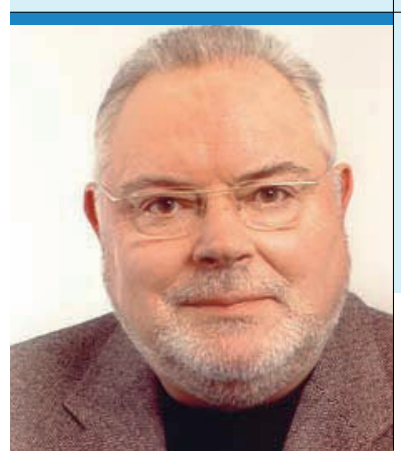

„Für Ihre Treue und den kontinuierlichen Zuspruch

über all die Jahre hinweg bedanken wir uns ganz

herzlich."

Dr. med. Dieter Leithäuser

Chefredakteur und niedergelassener HNO-Arzt, Warburg

\section{Jahre und ein stolzer Blick zurück}

S eit 1970 bestehen sie nun, die HNO Nachrichten. Was mit dem Slogan „liquidieren wird einfacher" begann, kann mittlerweile also seinen 40 . Geburtstag feiern. Eine lange Zeit für eine Zeitschrift und Grund genug für einen stolzen Blick zurück.

Neben GOÄ prägten damals auch Begriffe wie BMÄ, E-GO, KVB I-III und BG unseren Praxisalltag. Kollegen wie Gert Bienias haben mit der ersten Ausgabe der HNO-Nachrichten begonnen, Hilfestellung bei allen Abrechnungsfragen des HNOArztes zu geben. Keine leichte Aufgabe bei einem anfänglichen Heftvolumen von zwölf Seiten. Für Fortbildungsbeiträge war dabei nur wenig Platz, zu groß war die Zahl der Abrechnungsziffern. Böse Zungen behaupteten, der HNO-Arzt ließe sich jeden Handgriff einzeln bezahlen.

Nach dem Verlagswechsel vom Frühmorgen Verlag zu Urban \& Vogel erhielt die Zeitschrift ein neues Gesicht. Das Prinzip „von Kollegen für Kollegen" blieb erhalten. Hinzu kam jedoch eine professionelle Redaktion im Hintergrund. Und neue Technik hielt Einzug: Die häusliche Schreibmaschine machte dem damals noch sündhaft teuren Personal-Computer mit angegliedertem Fax Platz.

1995 übernahm Hartmut Sauer das Ruder in der Chefredaktion. Im gleichen Jahr begann meine Mitarbeit mit dem Serien-Beitrag: „Allergologie - aus der Praxis für die Praxis“. Die Abbildungen dazu wurden mit dem Programm Corel noch in SchwarzWeiß gezeichnet. Zur Jahrtausendwende wurde mir dann die Verantwortung in der Chefredaktion übertragen. Das Heft wurde bunter, endlich waren auch Farbabbildungen möglich. Ausgestattet mit einem schreibfreudigen, kompetenten Beirat aus Klinik und Praxis war mein Beitrag zu den HNONachrichten fast immer stressfrei. Die hauptamtlichen Redaktionsmitglieder wechselten turnusmäßig, aber immer waren sie alle mit großem Engagement dabei und auf gute Zusammenarbeit bedacht. Drei Umzüge an einen anderen Standort mussten überstanden werden, ebenso einige Wechsel in den Besitzverhältnissen der Verlagsgruppe.

So abwechslungsreich die Geschichte auch war, eines können wir Ihnen versichern: Auch in Zukunft werden wir, das Redaktionsteam von HNO-Nachrichten, Ihnen praxisnahe Informationen zu Berufspolitik, Praxismanagement, Recht und Finanzen liefern. Im Fortbildungsteil werden auch weiterhin hochkarätige Autoren aus dem klinischen Bereich und aus den Reihen unserer niedergelassenen Kollegen ihre Beiträge präsentieren. Nicht in epischer Breite, sondern dem knappen Zeitrahmen des in der Praxis stehenden Kollegen angepasst.

Feiern Sie mit uns unser vierzigjähriges Jubiläum. In München würde man dieses Ereignis mit den Worten starten: Ozapft is! Packen wir es gemeinsam an: Per aspera ad astera - auf rauen Pfaden zu den Sternen.

Ihr
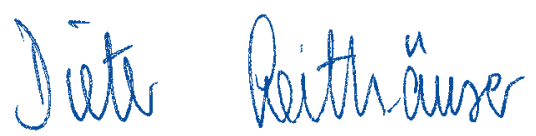